\title{
FACTORES ASOCIADOS A REPERCUSIONES PERINATALES DESFAVORABLES EN GESTANTES CON OLIGOHIDRAMNIOS EN EL HOSPITAL DE VITARTE, PERIODO 2016 - 2019
}

\author{
FACTORS ASSOCIATED WITH UNFAVORABLE PERINATAL IMPACTS IN \\ PREGNANTS WITH OLIGOHIDRAMNIOS IN VITARTE HOSPITAL, 2016-2019.
}

\author{
Gabriela T. Cotera-Abad ${ }^{1,2}$, Lucy E. Correa-Lopez ${ }^{1,3}$, Pedro M. Arango-Ochante ${ }^{2,3}$
}

\begin{abstract}
RESUMEN
Objetivos. Identificar los factores asociados a repercusiones perinatales desfavorables en gestantes con oligohidramnios en el hospital de Vitarte, periodo 2016 - 2019. Materiales y métodos. Se realizó un estudio analítico, retrospectivo, caso - control. La población de estudio fueron las gestantes con diagnóstico de oligohidramnios, por método ILA menor a $5 \mathrm{~cm}$, atendidas en el servicio de hospitalización de Ginecobstetricia en el hospital de Vitarte, durante el periodo 2016 - 2019; conformada por 97 casos y controles (1:1). Los casos fueron los que presentaron resultados perinatales desfavorables. En el análisis bivariado se utilizó la prueba de chi cuadrado, con un nivel de significancia de 0,05 . Se calcularon los OR con un Intervalo de confianza al $95 \%$ a través del programa SPSS. Resultados. Los neonatos con repercusiones desfavorables se presentaron en el $47 \%$. El tipo de parto más frecuente fue cesárea $(76 \%)$, evidenciándose asociación estadísticamente significativa con la resultante perinatal adversa $(O R=2,25 ; \quad I C 95 \%=1,21-4,19 ; p=0,009)$. No se encontró asociación con variables sociodemográficas maternas, inicio de parto, edad gestacional, número de controles prenatales, paridad, comorbilidad materna ni sexo del recién nacido. Conclusiones: La morbimortalidad perinatal es frecuente en gestantes con diagnóstico de oligohidramnios. Culminar el embarazo por cesárea es un factor asociado a repercusiones perinatales desfavorables. Además, la indicación de cesárea es innecesario solo por el hallazgo de oligohidramnios, ésta debe ser guiada por la patología subyacente.
\end{abstract}

Palabras clave. Oligohidramnios; Atención Perinatal; Recién Nacido (Fuente: DeCS BIREME).

\begin{abstract}
Objectives. Identify the factors associated with unfavorable perinatal repercussions in pregnant women with oligohydramnios at the hospital de Vitarte, period 2016-2019. Materials and methods. An analytical retrospective study of case and control was carried out. The population studied were pregnant women with diagnostic of oligohydramnios, by the ILA method of less than $5 \mathrm{~cm}$, attended at the Gynecology-Obstetrics hospitalization service at the hospital de Vitarte, during the period 2016-2019; made up of 97 cases and controls (1: 1). The cases were those that had unfavorable perinatal results. The chi-square test was used in the bivariate analysis, with a significance level of 0.05 . The ORs with a 95\% Confidence Interval were calculated through the SPSS program. Results. Newborns with unfavorable repercussions presented in the $47 \%$. The most frequent type of delivery was by caesarean section (76\%) and showing an association with the resulting adverse perinatal $(\mathrm{OR}=2.25 ; 95 \% \mathrm{Cl}=1.21$ - 4.19; $p=0.009$ ). No significant differences were found with maternal sociodemographic variables, start of labor, gestational age, number of prenatal controls, parity, maternal comorbidity and sex of the newborn. Conclusions. Perinatal morbidity and mortality is frequent in pregnant women diagnosed with oligohydramnios. Cesarean delivery is a factor associated with unfavorable perinatal repercussions. The indication for caesarean section is unnecessary only due to the finding of oligohydramnios, this should be guided by the underlying pathology.
\end{abstract}

Key words. Oligohydramnios; Prenatal Care; Newborn (Source: MeSH NLM).

\section{INTRODUCCIÓN}

El oligohidramnios es la disminución del volumen de líquido amniótico (LA), es decir un Índice de líquido amniótico menor a $5 \mathrm{~cm}$. El LA es un uno de los factores determinantes de la valoración del control prenatal ya que su alteración se asocia con un pobre pronóstico del embarazo. EI LAes esencial para el crecimiento y desarrollo fetal, así como para protegerlo de infecciones, amortiguar los traumatismos entre otros ${ }^{1-5}$.

El oligohidramnios está asociado a complicaciones maternas y neonatales, se observa en el $3-5 \%$ como

Facultad de Medicina Humana, Universidad Ricardo Palma. Lima-Perú

Servicio Gíneco-Obstetricia Hospital Vitarte. Lima-Perú.

Instituto de Investigación en Ciencias Biomédicas, Universidad Ricardo Palma, Lima - Perú.

Código ORCID: https://orcid.org/0000-0002-3267-1904, Pedro M. Arango Ochante

Citar como: Cotera-Abad GT, Correa-Lopez LE, Arango-Ochante PM. Factores asociados repercusiones perinatales desfavorables en gestantes con oligohidramnios en el Hospital de Vitarte, periodo 2016-2019. Rev Peru Investig Matern Perinat. 2021;10(1): 19-26 DOI https://doi.org/10.33421/inmp.2021225

Recibido: 22-01-2021 Aprobado: 31-03-2021 
factor que complica el embarazo ${ }^{2,6}$. A nivel mundial tiene una incidencia de 0,5 a $5 \%$ para los embarazos complicados por esta patología y es variable según la población estudiada y el método de diagnóstico ${ }^{(7)}$. En el Perú, según el INMP, se asocia de 10 a 15 veces más con la mortalidad perinatal, el cual aumenta de 40 a 50 veces más en el caso de oligohidramnios severo ${ }^{7,8}$.

La etología es múltiple, por alteraciones estructurales y anomalías congénitas del feto, insuficiencia placentaria, trastorno hipertensivo del embarazo, diabetes mellitus y ruptura prematura de las membranas. La fisiopatología más conocida es la disminución del flujo útero placentario, el cual reduce el flujo sanguíneo renal fetal y a su vez la producción de orina ${ }^{3,9}$.

El oligohidramnios es un factor de riesgo para complicaciones fetales y neonatales, así como indicador de posibles patologías maternas. Por ello, su diagnóstico debe orientar a una vigilancia estricta en el bienestar fetal $^{10}$. Las repercusiones perinatales adversas incluyen: score Apgar < 7 los 5 minutos, presencia de líquido meconial, estado fetal no tranquilizador, compresión del cordón umbilical, hipoxia y acidosis fetal, ingreso a unidades de cuidados intensivos neonatales, entre otros. Además limita el desarrollo del tejido pulmonar funcional, que lo llevará a tener problemas en su proceso de transición respiratoria en la vida extrauterina ${ }^{2,5}$.

El pronóstico perinatal es alarmante y aumenta 13 veces la morbimortalidad del recién nacido, en los casos de anhidramnios se asocia a 40 veces más este riesgo. Por lo tanto esta patología puede anticipar y alertar alguna situación que comprometa la vida del neonato ${ }^{1,2}$. No solo se debe conocer cuáles son las repercusiones perinatales adversas más frecuentes o graves, sino también que factores contribuyen para que el recién nacido presente alguna complicación. Actualmente no hay una conclusión exacta sobre estas repercusiones. Por lo cual el presente estudio pretende identificar los factores asociados a repercusiones perinatales desfavorables en gestantes con oligohidramnios en el hospital de Vitarte.

\section{MATERIALES Y MÉTODOS}

Se realizó un estudio observacional, analítico, retrospectivo de tipo caso - control. Se evaluó a todas las gestantes con diagnóstico de oligohidramnios atendidas en el servicio de hospitalización de Ginecobstetricia en el hospital de Vitarte durante el periodo Enero 2016 a Julio 2019. Las variables que se consideraron en el estudio fueron prenatales (edad gestacional, número de controles prenatales, paridad y comorbilidad materna), natales (inicio y tipo de parto) y posnatal (sexo del recién nacido). La clasificación de casos y controles fueron según los resultados perinatales, los casos fueron los que presentaron resultados desfavorables: score de
Apgar menor de 7 al primer y/o quinto minuto, pequeño o grande para la edad gestacional, bajo peso al nacer (menor de 2500gr) o macrosómico (mayor de 4000gr), presencia de líquido amniótico teñido con meconio, depresión moderada o severa, RCIU, alteración en la conducción cardiaca fetal, entre otras complicaciones del neonato o ingreso a la unidad de cuidados intermedios. Los controles fueron los que tuvieron resultados perinatales favorables. Se incluyeron aquellas que fueron diagnosticadas por método ILA menor de $5 \mathrm{~cm}$, embarazos a término y madres adolescentes y añosas (mayor de 35 años). Mientras que se excluyó historias clínicas incompletas, sin tarjeta de control prenatal, parto extra hospitalario, recién nacidos con malformaciones, diagnóstico de rotura de membrana o gestante referida a otro hospital de mayor complejidad.

Se encontró 348 gestantes con diagnóstico de oligohidramnios y se excluyeron 141 historias clínicas, teniendo como muestra final 207 pacientes. De los cuales 97 recién nacidos conformaron el grupo de estudio (casos) y 97 el grupo control, por muestreo aleatorio simple y emparejamiento por edad.

Se utilizó el programa estadístico IBM SPSS v.25 para el análisis estadístico, las variables cualitativas fueron descritas mediante frecuencias y porcentajes. En el análisis bivariado se utilizó la prueba de chi cuadrado con un nivel de significancia de 0,05. Se realizó el cálculo de razón de riesgo (Odss rattio) entre los casos y controles, así como sus respectivos intervalos de confianza al $95 \%$.

\section{RESULTADOS}

La frecuencia de repercusiones perinatales desfavorables producto de gestantes con oligohidramnios fue 47\% (97 casos), incluyendo un óbito fetal.

Los resultados descriptivos de los factores sociodemográficos maternos se describe en la Tabla 1. La edad promedio de las gestantes con oligohidramnios fue de 26,8 años, en su distribución la población más frecuente estuvieron entre 25 - 34 años (52\%). En la mayoría de los casos se dedicaban al hogar (89\%), estaban en condición de conviviente $(81 \%)$ y el grado de instrucción más frecuente fue el nivel de escolaridad secundaria $(69 \%)$.

En el análisis bivariado estos factores sociodemográficos no presentaron asociación estadísticamente significativa con las repercusiones perinatales desfavorables (Tabla 2).

En la Tabla 3 se presenta los resultados del análisis de las variables prenatales, natales y postnatal con las repercusiones perinatales desfavorables. En el grupo de neonatos con repercusiones perinatales adversas, el 
Tabla 1. Factores sociodemográficos maternas y repercusiones perinatales desfavorables en gestantes con oligohidramnios en el hospital de Vitarte, periodo $2016-2019$.

\begin{tabular}{ccccc}
\hline $\begin{array}{c}\text { Factores } \\
\text { sociodemográficos } \\
\text { maternos }\end{array}$ & $\begin{array}{c}\text { RPD } \\
\text { (n=97) }\end{array}$ & $\begin{array}{c}\text { RPF } \\
(\mathbf{n = 9 7 )}\end{array}$ \\
\hline Edad materna & 35 & $36 \%$ & 42 & $43 \%$ \\
\hline $15-24$ años & 50 & $52 \%$ & 42 & $43 \%$ \\
\hline $25-34$ años & 12 & $12 \%$ & 13 & $13 \%$ \\
\hline$\geq 35$ años & 7 & $7 \%$ & 6 & $6 \%$ \\
\hline Estado civil & 79 & $81 \%$ & 84 & $87 \%$ \\
\hline Casada & 11 & $11 \%$ & 7 & $7 \%$ \\
\hline Conviviente & & & & \\
\hline Soltera & 86 & $89 \%$ & 85 & $88 \%$ \\
\hline Ocupación & 2 & $2 \%$ & 4 & $4 \%$ \\
\hline Ama de casa & 9 & $9 \%$ & 8 & $8 \%$ \\
\hline Profesional & & & & \\
\hline Independiente & 6 & $6 \%$ & 12 & $12 \%$ \\
\hline Grado de instrucción & 67 & $69 \%$ & 60 & $62 \%$ \\
\hline Primaria & 24 & $25 \%$ & 25 & $26 \%$ \\
\hline Secundaria & & & \\
\hline Superior & & & & \\
\hline
\end{tabular}

*RPD: Repercusiones Perinatales Desfavorables

${ }^{*}$ RPF: Repercusiones Perinatales Favorables

$92 \%$ presentaron edad gestacional mayor o igual a 37 semanas y solo el $8 \%$ mayor de 41 semanas, es decir producto de gestantes a término tardío. Además, $82 \%$ de las gestantes del grupo en estudio tuvieron en su mayoría un adecuado control prenatal. Según la paridad, se obtuvo que la mayoría de las gestantes en estudio fueron nulíparas (53\%). En cambio, en las gestantes del grupo control se obtuvo porcentajes contrarios, es decir fueron más frecuente las que tuvieron más de un hijo (57\%). No se halló asociación estadísticamente significativa entre edad gestacional, número de controles prenatales y paridad con repercusiones perinatales adversas.

Con respecto a las comorbilidades maternas encontradas en el grupo de estudio, más frecuentes fueron infección del tracto urinario $(35 \%)$, anemia (29\%) y distocias (15\%). Además, en la Tabla 4, se resume la frecuencia de las patologías encontradas en las gestantes con oligohidramnios y las repercusiones perinatales. En ambos grupos se encontró similitud entre las enfermedades más frecuentes, es decir ITU, anemia y distocia. Pero en los trastornos hipertensivos del embarazo y hemorragia del III trimestre se evidenció más casos en los neonatos que presentaron repercusiones perinatales desfavorables ( $11 \%$ y $6 \%$ respectivamente). Las patologías maternas se presentaron mayormente en el grupo control $(61 \%)$ pero sin marcada diferencia. Al realizar el análisis bivariado entre la presencia de comorbilidad materna y resultante perinatal adversa en gestantes con oligohidramnios no se encontró asociación estadísticamente significativa $(p=0,661 ;$ IC95\%=0,49 - 1,56\%), (Tabla 3).

En relación a las variables natales, la vía de culminación del embarazo en gestantes con repercusión perinatal adversa se observó más frecuente el parto por cesárea (76\%), mientras que el $24 \%$ fue parto eutócico; de las cuales $56 \%$ fueron inducidos y $43 \%$ espontáneos. En las gestantes cuyos recién nacidos no presentaron alguna repercusión, la vía de parto más frecuente también fue cesárea (59\%), (Tabla 3). La indicación más frecuente de cesárea en el grupo de estudio fue el estado fetal no tranquilizador (36\%), oligohidramnios aislado (24\%) y distocias (15\%), tal como se muestra en la Tabla 5.

Se obtuvo asociación estadísticamente significativa $\left(x^{2}=6,793 ; \quad p=0,009\right)$ entre parto por cesárea y repercusiones perinatales desfavorables (OR=2,25; IC95\%=1,21-4,19). En cambio, no se halló asociación con el inicio de parto, (Tabla 3).

Tabla 2. Asociación entre factores sociodemográficos maternos y repercusiones perinatales desfavorables en gestantes con oligohidramnios en el Hospital Vitarte, periodo 2016 - 2019.

\begin{tabular}{|c|c|c|c|c|c|c|c|c|}
\hline \multirow{2}{*}{$\begin{array}{c}\text { Factores sociodemográficos } \\
\text { maternos }\end{array}$} & \multicolumn{2}{|c|}{ RPD } & \multicolumn{2}{|c|}{ RPF } & \multirow{2}{*}{ Chi Cuadrado } & \multirow{2}{*}{$\begin{array}{l}\text { p va } \\
\text { lor }\end{array}$} & \multirow{2}{*}{ OR crudo } & \multirow{2}{*}{ IC $95 \%$ OR } \\
\hline & $\mathrm{n}$ & $\%$ & $\mathrm{n}$ & $\%$ & & & & \\
\hline \multicolumn{9}{|l|}{ Edad materna } \\
\hline$\leq 180 \geq 35$ años & 18 & $19 \%$ & 19 & $20 \%$ & & & 0,93 & $0,45-1,91$ \\
\hline $19-34$ años ${ }^{*}$ & 79 & $81 \%$ & 78 & $80 \%$ & 0,033 & 0,855 & 1,00 & \\
\hline \multicolumn{9}{|l|}{ Estado civil } \\
\hline Sin pareja & 11 & $11 \%$ & 7 & $7 \%$ & 0,980 & 0,322 & 1,64 & $0,60-4,43$ \\
\hline Con pareja* & 86 & $89 \%$ & 90 & $93 \%$ & & & 1,00 & \\
\hline \multicolumn{9}{|l|}{ Ocupación } \\
\hline Ama de casa & 86 & $89 \%$ & 85 & $88 \%$ & 0,049 & 0,824 & 1,10 & $0,46-2,63$ \\
\hline Profesional $^{*}$ & 11 & $11 \%$ & 12 & $12 \%$ & & & 1,00 & \\
\hline \multicolumn{9}{|l|}{ Grado de instrucción } \\
\hline No superior & 73 & $75 \%$ & 72 & $74 \%$ & 0,027 & 0,869 & 1,05 & $0,55-2,01$ \\
\hline Superior* & 24 & $25 \%$ & 25 & $26 \%$ & & & 1,00 & \\
\hline
\end{tabular}

*Categoría de referencia

${ }^{*}$ RPD: Repercusiones Perinatales Desfavorables

${ }^{*}$ RPF: Repercusiones Perinatales Favorables 
Tabla 3. Análisis bivariado de las variables y repercusiones perinatales desfavorables en gestantes con oligohidramnios en el Hospital Vitarte en el periodo 2016 - 2019.

\begin{tabular}{|c|c|c|c|c|c|c|c|c|}
\hline VARIABLES & \multicolumn{2}{|c|}{ RPD } & \multicolumn{2}{|c|}{ RPF } & Chi Cuadrado & \multirow{2}{*}{ p valor } & \multirow{2}{*}{ OR crudo } & \multirow{2}{*}{ IC $95 \%$ OR } \\
\hline \multicolumn{6}{|l|}{ V. PRENATALES } & & & \\
\hline \multicolumn{9}{|l|}{ Edad Gestacional } \\
\hline$>41$ semanas & 8 & $8 \%$ & 5 & $5 \%$ & & & 1,65 & $0,51-5,24$ \\
\hline$\geq 37$ semanas* & 89 & $92 \%$ & 92 & $95 \%$ & 0,742 & 0,389 & 1,00 & \\
\hline \multicolumn{9}{|c|}{ Número de controles prenatales } \\
\hline Insatisfactorio (<6) & 17 & $18 \%$ & 31 & $32 \%$ & & & 0,45 & $0,23-0,88$ \\
\hline Satisfactorio $(\geq 6)^{*}$ & 80 & $82 \%$ & 66 & $68 \%$ & 5,426 & 0,020 & 1,00 & \\
\hline \multicolumn{9}{|l|}{ Paridad } \\
\hline Nulípara & 51 & $53 \%$ & 42 & $43 \%$ & & & 1,45 & $0,82-2,55$ \\
\hline Primípara / Multípara* & 46 & $47 \%$ & 55 & $57 \%$ & 1,673 & 0,196 & 1,00 & \\
\hline \multicolumn{9}{|l|}{ Comorbilidad materna } \\
\hline $\mathrm{Si}$ & 56 & $58 \%$ & 59 & $61 \%$ & & & 0,88 & $0,49-1,56$ \\
\hline $\mathrm{No}^{*}$ & 41 & $42 \%$ & 38 & $39 \%$ & 0,192 & 0,661 & 1,00 & \\
\hline \multicolumn{9}{|l|}{ V. NATALES } \\
\hline \multicolumn{9}{|l|}{ Tipo de parto } \\
\hline Cesárea & 74 & $76 \%$ & 57 & $59 \%$ & & & 2,25 & $1,21-4,19$ \\
\hline Vaginal* $^{*}$ & 23 & $24 \%$ & 40 & $41 \%$ & 6,793 & 0,009 & 1,00 & \\
\hline \multicolumn{9}{|l|}{ Inicio de parto } \\
\hline Inducido & 54 & $56 \%$ & 51 & $53 \%$ & & & 1,13 & $0,64-1,99$ \\
\hline Espontáneo* & 43 & $44 \%$ & 46 & $47 \%$ & 0,187 & 0,666 & 1,00 & \\
\hline \multicolumn{9}{|l|}{ V. POSTNATAL } \\
\hline \multicolumn{9}{|l|}{ Sexo del recién nacido } \\
\hline Masculino & 56 & $58 \%$ & 49 & $51 \%$ & & & 1,33 & $0,75-2,35$ \\
\hline Femenino* & 41 & $42 \%$ & 48 & $49 \%$ & 1,017 & 0,313 & 1,00 & \\
\hline
\end{tabular}

${ }^{*}$ Categoría de referencia

${ }^{*}$ RPD: Repercusiones Perinatales Desfavorables

*RPF: Repercusiones Perinatales Favorables

En relación a la variable posnatal, sexo del recién nacido, el más frecuente fue el sexo masculino (58\%), el cual no se evidenció asociación estadísticamente significativa con la resultante perinatal adversa (Tabla 3 ).

Tabla 4. Estadística descriptiva de las comorbilidades maternas y repercusiones perinatales en gestantes con oligohidramnios en el Hospital Vitarte en el periodo 2016 $-2019$

\begin{tabular}{|c|c|c|c|c|}
\hline \multirow{2}{*}{$\begin{array}{c}\begin{array}{c}\text { COMORBILIDAD } \\
\text { MATERNA }\end{array} \\
\text { Anemia }\end{array}$} & \multicolumn{2}{|c|}{ RPD } & \multicolumn{2}{|c|}{ RPF } \\
\hline & 21 & $29 \%$ & 32 & $41 \%$ \\
\hline THE & 8 & $11 \%$ & 3 & $4 \%$ \\
\hline ITU & 25 & $35 \%$ & 28 & $35 \%$ \\
\hline IUP & 3 & $4 \%$ & 2 & $3 \%$ \\
\hline Hemorragia III TRI & 4 & $6 \%$ & 2 & $3 \%$ \\
\hline Distocias & 11 & $15 \%$ & 12 & $15 \%$ \\
\hline
\end{tabular}

*THE: Trastorno Hipertensivo del Embarazo.

*ITU: Infección del Tracto Urinario

*IUP: Insuficiencia Útero Placentaria

${ }^{*}$ RPD: Repercusiones Perinatales Desfavorables

*RPF: Repercusiones Perinatales Favorables
Tabla 5. Frecuencia de indicaciones de cesárea en las gestantes con oligohidramnios en el hospital Vitarte en el periodo 2016 - 2019.

\begin{tabular}{lcccc}
\hline \multirow{2}{*}{ INDICACIÓN DE CESÁREA } & \multicolumn{2}{c}{ RPD } & \multicolumn{2}{l}{ RPF } \\
\cline { 2 - 5 } & \multicolumn{3}{c}{ RP } \\
\hline Estado fetal no tranquilizador & 27 & $36 \%$ & 0 & $0 \%$ \\
Oligohidramnios aislado & 18 & $24 \%$ & 40 & $70 \%$ \\
\hline Distocias & 11 & $15 \%$ & 12 & $21 \%$ \\
Macrosomía & 7 & $9 \%$ & 0 & $0 \%$ \\
THE & 5 & $7 \%$ & 2 & $4 \%$ \\
IUP & 2 & $3 \%$ & 2 & $4 \%$ \\
Hemorragia III TRI & 4 & $5 \%$ & 1 & $2 \%$ \\
\hline
\end{tabular}

*THE: Trastorno Hipertensivo del Embarazo.

*IUP: Insuficiencia Útero Placentaria

${ }^{*}$ RPD: Repercusiones Perinatales Desfavorables

${ }^{\star}$ RPF: Repercusiones Perinatales Favorables

\section{DISCUSIÓN}

En múltiples estudios se evidencia que el oligohidramnios es un factor de riesgo para presentar repercusiones perinatales desfavorables; el pronóstico perinatal es preocupante en estos casos y se ha descrito que aumenta 
13 veces la morbimortalidad perinatal $\left.\right|^{(1,11-13)}$. El enfoque de este estudio fue conocer qué factores intervienen en el resultado perinatal adverso asociado a oligohidramnios.

En el estudio se encontró que el $47 \%$ de neonatos producto de gestantes a término con oligohidramnios presentaron repercusiones desfavorables. Similar a lo reportado en un estudio realizado en el Hospital Nacional Hipólito Unánue con un 52,3\%, incluyendo embarazos pre término y post término ${ }^{14}$. Sin embardo, en el estudio solo se halló un óbito fetal, menor a lo reportado en el estudio del Hospital Nacional Daniel Alcides Carrión con $4 \%$ de mortalidad $^{15}$. Por lo que se refiere que la morbimortalidad perinatal no es infrecuente.

Con respecto a la edad materna se encontró que la edad promedio fue de 26,8 años y en general era más frecuente en mujeres entre 25-34 años. Tal como en el estudio del HNHU, edad promedio de 27,4 años y grupo más afectado entre 25-35 años ${ }^{14}$. La edad materna en esta investigación incluyó a pacientes añosas y adolescentes, consideradas como gestantes de alto riesgo; sin embargo, no se evidenció asociación como factor de riesgo para que presente alguna repercusión en el recién nacido.

Las determinantes sociodemográficas materna más frecuentes fueron mujeres convivientes, amas de casa y de grado de instrucción inferior; los cuales se asemejan a la frecuencia reportada en estudios de un hospital de México y del hospital de Maternidad de Lima ${ }^{2,4}$. Al realizar el análisis de estado civil, ocupación y grado de instrucción como factor de riesgo no se encontraron asociación con repercusiones perinatales desfavorables. Además, los resultados de estas variables fueron de frecuencia similar entre casos y controles por lo que al no encontrase asociación se puede inferir que no tienen relación en la aparición de repercusiones desfavorables en el neonato, aunque en el estudio de Gonzales G; refiere que un grado de instrucción inferior podría influir en el incumplimiento de las indicaciones médicas en gestantes con oligohidramnios ${ }^{4}$.

En cuanto a las variables prenatales, no se encontró asociación entre edad gestacional con repercusiones perinatal desfavorables y se halló más frecuente en mayor o igual a 37 semanas (92\%), es decir en el embarazo a término temprano (37-38 6/7) y a término completo (39-40 6/7) en comparación con el término tardío (41-41 6/7), tal como se reportó en el estudio de Karahanoglu $E^{9}$ en el cual, además; refiere que las repercusiones neonatales adversas no fueron uniformes en los periodos del embarazo a término, siendo mayor en el a término tardío, el cual no se reportó en este estudio posiblemente porque se usaron menos parámetros que incluyeron los casos de estudio.

Con respecto a la paridad, se halló más frecuente en nulíparas pero sin asociación como factor de riesgo. En los artículos revisados, no hubo información disponible con respecto a dicha asociación, sin embargo; un estudio de Honduras asocia las primigestas con mayor riesgo de oligohidramnios moderado ${ }^{16}$. Por lo que se infiere que pese a ser frecuente en el grupo de estudio no es factor de riesgo para afectar la resultante perinatal.

El adecuado control prenatal se presentó en la mayoría de pacientes del estudio, resultado que difiere del estudio del $\mathrm{HNHU}^{14}$ el cual reportó que fue mayor los controles prenatales insatisfactorios. Además, no se demostró asociación de esta variable con resultados perinatales adversos.

Las comorbilidades maternas más frecuentes según estudio del $\mathrm{HNHU}^{14}$ fueron en el 21,5\% RPM, 18,7\% anemia, $16,8 \%$ ITU y $14 \%$ preeclamsia. Otro estudio del Hospital de la Maternidad ${ }^{4}$ refiere que más frecuente fue anemia materna (12\%) y trastornos hipertensivos del embarazo $(7,4 \%)$. Sin embargo, en el estudio fueron: infección del tracto urinario (35\%), anemia (29\%) y distocias (15\%), pero al hacer el análisis no hubo asociación entre patología materna y repercusión perinatal desfavorable. Además, éstos fueron similares en ambos grupos de estudio y como esta descrito en la literatura no están relacionadas como etiologías de oligohidramnios, lo cual explicaría la falta de asociación. Tal como refiere la investigación de Rivas Perdomo $\mathrm{E}^{17}$; la anemia materna no se asoció con resultados perinatales adversos, pero que se podría dar en casos de anemia severa (hb menor de $6 \mathrm{~g} / \mathrm{dl}$ ). En el estudio de $\mathrm{USA}^{18}$, describe que embarazos con oligohidramnios sin comorbilidades presentan ciertos resultados neonatales adversos, mientras en las gestantes con comorbilidades si hay asociación, aunque probablemente se deba más por la condición mórbida o etiología del oligohidramnios que está en sí misma, como preeclamsia e insuficiencia útero placentaria.

En la investigación de Pérez $L^{19}$; refiere que un porcentaje de las gestantes (abarcando toda edad gestacional) con insuficiencia placentaria leve se asociaron a morbimortalidad perinatal, debido a una invasión anormal y superficial de trofoblastos en la vascularización materna, generando aumento de la resistencia al flujo e intercambio deficiente materno fetal; posteriormente produce redistribución hemodinámica y por hipoperfusión renal conlleva a oligohidramnios. Además, señala que la ecografía Doppler de la arteria uterina sería el mejor predictor de repercusión perinatal adversa. En este estudio las patologías más frecuentes relacionadas como etiología del oligohidramnios en gestantes a término fueron Trastornos hipertensivos del embarazo y hemorragia del III trimestre, siendo mayores en el grupo de estudio pero sin diferencia significativa ni asociación, incluyendo los casos de insuficiencia placentaria.

La inducción del trabajo de parto, según algunos estudios $^{5,20}$, es más frecuente que el espontáneo en el manejo de la gestante con oligohidramnios, tal como se contrasta con los resultados obtenidos en esta 
investigación; sin embargo no se demostró asociación como factor de riesgo para repercusiones perinatales adversas. Según la ACOG la inducción del trabajo de parto en gestantes con oligohidramnios mayor de 37 semanas, siempre y cuando no haya empezado el parto espontáneo ni tampoco se evidencie compromiso materno o fetal. En dos estudios realizados en Turquía e Israel reportaron que el parto inducido fue el más frecuente tanto en oligohidramnios aislado como en los que presentaron comorbilidades maternas ${ }^{9,21}$, además; en esta investigación esta indicación fue más frecuente en ambos grupos de gestantes con oligohidramnios independiente de la repercusión perinatal. Por lo cual se infiere que el inicio de parto no interviene en la resultante perinatal y es frecuente en el manejo de esta patología. Sin embargo, a pesar que sea la indicación más adecuada no se tiene la certeza que culminarán por vía vaginal, ya que en el estudio de Bedoya M. en el HNDAC la mayoría de gestantes con oligohidramnios que fueron inducidas terminaron por cesárea ${ }^{15}$

En múltiples estudios nacional e internacionales $2,5,7,12-14$ se ha evidenciado el parto por cesárea como la vía de culminación más frecuente en gestantes con oligohidramnios, tanto en casos de oligohidramnios aislado como en aquellos con comorbilidades maternas. Lo cual también se evidenció en esta investigación, siendo la cesárea mayor en ambos grupos pero con diferencia significativa y superior en aquellos con repercusiones perinatales desfavorables. Por lo cual también se demostró asociación como factor de riesgo entre estas variables, aunque no se ha encontrado literatura con la cual se pueda contrastar porque tuvieron una población de estudio o método diferente.

La indicación de cesárea más frecuente reportado en otros estudios es el estado fetal no tranquilizador ${ }^{9,22}$, tal como lo encontrado en esta investigación. Se ha descrito en la literatura que esta complicación perinatal se debe a la compresión del cordón umbilical durante el parto, por la insuficiencia placentaria sutil de difícil diagnostico por las técnicas actuales disponibles y por los casos de neonatos pequeños para la edad gestacional sin diagnóstico previo ${ }^{5,9}$. Además, esta complicación neonatal no traduce hipoxia, pero indica el riesgo de presentarla y posteriormente lesión cerebral ${ }^{23}$. En el estudio del HNDAC refiere que existe relación entre el CST alterado y el estado fetal no tranquilizador con la severidad del oligohidramnios ${ }^{15}$. Por ello se sugiere la adecuada monitorización fetal y uso de pruebas de bienestar fetal.

La vía de culminación del embarazo a término más adecuada es controversial. En Alemania según Hollwtiz M; reportó que el parto vaginal fue más frecuente y refiere la importancia del uso de la ecografía Doppler en los fetos con peso menor o igual a percentil 10 para que garantice la seguridad del parto vaginal, además que esta ecografía con hallazgo patológico es un factor de riesgo más fuerte que el oligohidramnios para tener neonatos con repercusión adversa $^{22}$. En el estudio del Hospital de la Maternidad de Lima también encontró que la mayoría de gestantes a término con oligohidramnios tuvieron parto vaginal y de inicio espontáneo(4). Mientras que otros estudios reportan que el parto por cesárea fue más frecuente, aunque no lo recomiendan como la indicación más adecuada; según la investigación de Rabie $\mathrm{N}$. en USA ${ }^{18}$, sugiere que en los embarazos con oligohidramnios de alto riesgo o con comorbilidades maternas debe guiarse la elección de parto por cesárea por la comorbilidad materna y no solo por el oligohidramnios. Otro estudio en Venezuela ${ }^{5}$ señala que no hay mayor beneficio en la repercusión perinatal ni a corto o largo plazo por la interrupción inmediata en casos de oligohidramnios aislados; además, existe la posibilidad que aumente las complicaciones neonatales por cesárea.

Según estudio de Italia ${ }^{7}$, indica más frecuente el parto por cesárea sin presentar repercusiones perinatales desfavorables en gestantes con oligohidramnios aislado. Además refiere que las intervenciones quirúrgicas por oligohidramnios podrían ser por la política institucional o actitud de obstetras. Así mismo, en el estudio de Ardilla $\mathrm{V}^{5}$; se ha descrito que por la ausencia de una política específica institucional más de la mitad de gestantes de bajo riesgo (sin comorbilidades maternas) con oligohidramnios aislado fueron a cesárea, además que esta decisión podría estar asociado más con el temor de los médicos por las complicaciones que por el oligohidramnios. Tal como se contrasta con los resultados de esta investigación, ya que la indicación de cesárea por oligohidramnios aislado fue superior en los recién nacidos que no presentaron repercusiones desfavorables.

El único que recomendó el parto por cesárea fue un estudio de México $^{2}$, porque refiere que el parto vaginal expone al feto a estrés durante el trabajo de parto; además, contribuye la cesárea a la ausencia de repercusiones perinatales adversas producto de gestantes con oligohidramnios aislado severo. Sin embargo, la razón de cesárea fue esta patología y no por sufrimiento fetal. Por lo tanto, es importante el uso de pruebas de bienestar fetal y estudio Doppler, si lo amerita, para la elección de la vía de culminación del embarazo. Además, pese a ser el parto por cesárea el más frecuente y porque se ha demostrado en esta investigación que es un factor de riesgo para las repercusiones adversas en los recién nacidos, más que una consecuencia de ello; se debería evaluar la indicación de cesárea según la condición de la gestante y del feto, y no guiarse solo por el diagnóstico de oligohidramnios.

En el manejo de la gestante con oligohidramnios, un estudio de Cuba señala que el uso de hidroterapia materno endovenosa permitió tener mayor cantidad de partos por vía vaginal de manera espontánea y menos casos de estado fetal no tranquilizante $u$ otras complicaciones neonatales por las cuales se indica cesárea; además, que fue seguro y bien tolerado ${ }^{1,18}$. Por lo cual un buen manejo en la gestante y adecuado monitoreo fetal permitiría terminar el embarazo por parto vaginal. 
En el estudio fue más frecuente el sexo masculino, así como en la investigación de México ${ }^{2}$ donde se reportó en el $59,4 \%$ y en Cuba $^{16}$ con un $54,1 \%$; éste además refiere asociación entre el sexo masculino y oligohidramnios. Sin embargo, en el estudio no se evidenció asociación con las repercusiones perinatales adversas y tampoco se encontró literatura que contraste dicho resultado.

En conclusión, la morbimortalidad perinatal es frecuente en las gestantes con diagnóstico de oligohidramnios. Las variables sociodemográficas maternas, prenatales y posnatal no presentaron asociación estadísticamente significativa con la resultante perinatal adversa. La elección de culminar el embarazo por cesárea es un factor de riesgo asociado a repercusiones perinatales desfavorables, siendo la indicación más frecuente hallada el estado fetal no tranquilizador. Además, la indicación de cesárea para la interrupción inmediata del embarazo es innecesario solo por el hallazgo de diagnóstico de oligohidramnios, ésta debe ser guiada por la patología subyacente.

\section{RECOMENDACIONES}

Se recomienda un adecuado monitoreo fetal y uso de pruebas de bienestar fetal para garantizar la seguridad del parto y detectar oportunamente el diagnostico de estado fetal no tranquilizador, ya que se ha descrito relación entre esta complicación fetal con la severidad del oligohidramnios ${ }^{15}$.Se recomienda que la vía de culminación del embarazo más adecuada es por parto vaginal, siempre y cuando la condición materna y fetal lo permita. Priorizar el adecuado manejo de hidratación de la gestante con oligohidramnios para evitar complicaciones neonatales y aumento de cesáreas por éstas ${ }^{1}$.

Por último se sugiere ampliar el estudio con más parámetros de repercusiones neonatales desfavorables y su relación con los periodos de embarazo a término. Así como dar continuidad con un estudio prospectivo sobre el manejo de la hidratación materna y parto por cesárea, en una mayor población de estudio que permitirá obtener mejor poder estadístico y con método de diagnóstico de bolsa vertical, ya que produce menos falsos positivos.

Declaración de conflictos de intereses. Los autores declaran no tener conflicto de intereses.

Financiamiento. Autofinanciado.

\section{REFERENCIAS BIBLIOGRÁFICAS}

1. Amador-de-Varona $\mathrm{Cl}$, Cabrera-Figueredo I, RodríguezFernández JM, Valdés-Dacal S, Niño-Victoria Y, Nieves-Martínez J. Hidroterapia materna endovenosa en el oligohidramnios. Rev. Arch Méd Camagüey [Internet]. 2019; 23(1):85-94.

2. Ulloa KLG, Panduro-Barón JG, Camarena-Pulido EE, Quintero-Estrella IM, Barrios-Prieto E, Fajardo-Dueñas S.
Repercusiones perinatales en embarazos a término con oligohidramnios severo. Rev. Médica MD [Internet]. 2013; 5(4):245-50.

3. Cunningham GF W. Obstetricia. 24․ McGraw-Hill. 1350; 2015.

4. González Guidos EAJ. Resultado perinatal de embarazos a término con oligohidramnios de moderado a severo que consultan en el Hospital Nacional de Maternidad "Dr. Raúl Arguello Escolán" entre el periodo de enero a diciembre de 2013 [Internet]. 2013. Disponible en: http://ri.ues.edu.sv/id/ eprint/9958/1/Tesis.pdf

5. Ardila-Villa F, Villasmil ER, Cepeda DT, Montilla JM, Villasmil $\mathrm{NR}$, Ramírez AF, et al. Oligohidramnios aislado y resultante neonatal en embarazos a término. Av. En Biomed [Internet]. 16 de octubre de 2017; 6(2):113-9. Disponible en: http://erevistas. saber.ula.ve/index.php/biomedicina/article/view/9374

6. T. Dulay A. Oligohidramnios - Ginecología y obstetricia [Internet]. Manual MSD versión para profesionales. 2019.

7. Rossi AC, Prefumo F. Perinatal outcomes of isolated oligohydramnios at term and post-term pregnancy: a systematic review of literature with meta-analysis. Eur $\mathrm{J}$ Obstet Gynecol Reprod Biol. [Internet]. 1 de julio de 2013; 169(2):149-54. Disponible en: https://www.ejog.org/article/ S0301-2115(13)00139-5/abstract

8. Guías de práctica clínica y de procedimientos en obstetricia y perinatología. En: Instituto Nacional Materno Perinatal [Internet]. 2. Lima, Perú; 2018. p. 536.

9. Karahanoglu E, Akpinar F, Demirdag E, Yerebasmaz N, Ensari T, Akyol A, et al. Obstetric outcomes of isolated oligohydramnios during early-term, full-term and late-term periods and determination of optimal timing of delivery. J Obstet Gynaecol Res [Internet]. 2016; 42(9):1119-24. Disponible en: https://obgyn.onlinelibrary.wiley.com/doi/ abs/10.1111/jog.13024

10. Jiménez García, A, Romeu Martínez M, Vázquez Lara D. Manual básico de Obstetricia y Ginecología [Internet]. 364; 388 p. (Capítulo 12.Cuidados iniciales al recién nacido).

11. Hesson A, Langen E. Outcomes in oligohydramnios: the role of etiology in predicting pulmonary morbidity/mortality. $\mathrm{J}$ Perinat Med. 25 de octubre de 2018; 46(8):948-50.

12. Quispe Serna M, Rocha Torres KI. "Complicaciones perinatales en gestantes con oligohidramnios. Hospital regional de Ayacucho. Septiembre - noviembre 2016". Univ. Nac San Cristóbal Huamanga [Internet]. 2016; Disponible en: http://repositorio.unsch.edu.pe/handle/UNSCH/1862

13. Vicerrel $\mathrm{C}$, Jesús $\mathrm{T}$ de. Factores perinatales asociados a oligohidramnios en gestantes atendidas en el Hospital Nacional Docente Madre Niño - San Bartolomé en el periodo junio 2010-mayo 2011. 2014; 56-56. Disponible en: http://cybertesis. unmsm.edu.pe/bitstream/cybertesis/3900/1/Cordova_vt.pdf

14. Soplin Vargas E. Hijo de madre con oligohidramnios, prevalencia y factores perinatales asociados en el Hospital Nacional Hipólito Unánue 2017. Univ. Nac. Federico Villarreal [Internet]. 2018 [citado 2 de julio de 2020]; Disponible en: http://repositorio.unfv.edu.pe/handle/UNFV/1760

15. Bedoya M, Carlos R. Resultados perinatales en gestantes de 38 a 41 sem. con oligohidramnios inducidas con oxitocina HNDAC enero 2005 - diciembre 2009. Repos Tesis - UNMSM [Internet]. 2013; Disponible en: http://cybertesis.unmsm.edu. pe/handle/20.500.12672/9909

16. Ochoa Fletes CA, Moreno F. Resultados perinatales adversos en pacientes en quienes se diagnosticó oligohidramnios en el 
Hospital Escuela. Rev. Médica Los Post Grados Med. 2008; 11(1):61-2.

17. Perdomo EER, Ciodaro CM. Estado ácido-base en recién nacidos de embarazo con oligohidramnios espontáneo. MedUNAB [Internet]. 2008; 11(2):103-6. Disponible en: https:// revistas.unab.edu.co/index.php/medunab/article/view/69

18. Rabie N, Meganne E, Steelman S, Ounpraseuth S. Oligohydramnios in complicated and uncomplicated pregnancy: a systematic review and meta-analysis. Ultrasound Obstet Gynecol Off J Int. Soc. Ultrasound Obstet Gynecol. 2017; 49(4):442-9.

19. Pérez Burrel L, Rasero B. Obstetric and perinatal outcomes in mild placental insufficiency. 2018. 77(6):373-80.

20. Balestena Sánchez JM, Almeida García G, Balestena Sánchez SG. Resultados del oligohidramnios en el parto y el recién nacido: Análisis caso-control. Rev. Cuba Obstet Ginecol [Internet]. abril de 2005; 31(1):0-0.

21. Shrem G, Nagawkar SS, Hallak M, Walfisch A. Isolated Oligohydramnios at Term as an Indication for Labor Induction: A Systematic Review and Meta-Analysis. Fetal Diana Ther. 2016; 40(3):161-73.
22. Hollwitz BG, Sousa MT de, Ortmeyer G, Hecher K. EP14.02: The role of oligohydraminos in delivery planning of small breech babies at term. Ultrasound Obstet Gynecol [Internet]. 2018; 52(S1):253-253. Disponible en: https://obgyn. onlinelibrary.wiley.com/doi/abs/10.1002/uog.19996

23. Méndez DN, Padrón MP. Fundamentaciones fisiopatológicas sobre la asfixia en el periparto. MEDISAN [Internet]. 2014; 18(3):401-15. Disponible en: https://www.redalyc.org/articulo. oa?id=368445003014

\section{Correspondencia:}

Gabriela T. Cotera-Abad

Dirección: Mz. A Lote 4. Asoc. Viv. Las terrazas. Sta. Clara, Lima, PERÚ

E-mail: gaaxxcc@gmail.com

Teléfono: 930658872 